\title{
Prolonged-Release Granules in Sachet
}

National Cancer Institute

\section{Source}

National Cancer Institute. Prolonged-Release Granules in Sachet. NCI Thesaurus. Code C149875.

Medicinal product consisting of prolonged-release granules presented in a sachet. 